\title{
Lusioersily
}

\section{Loss of Prolyl Hydroxylase-1 Protects Against Colitis Through Reduced Epithelial Cell Apoptosis and Increased Barrier Function}

Tambuwala, M., Cummins, E. P., Lenihan, C. R., Kiss, J., Stauch, M., Scholz, C. C., Fraisl, P., Lasitschka, F., Mollenhauer, M., Saunders, S. P., Maxwell, P. H., Carmeliet, P., Fallon, P. G., Schneider, M., \& Taylor, C. T. (2010). Loss of Prolyl Hydroxylase-1 Protects Against Colitis Through Reduced Epithelial Cell Apoptosis and Increased Barrier Function. Gastroenterology, 139(6), 2093. https://doi.org/10.1053/j.gastro.2010.06.068

Link to publication record in Ulster University Research Portal

Published in:

Gastroenterology

Publication Status:

Published (in print/issue): 03/12/2010

DOI:

10.1053/j.gastro.2010.06.068

\section{Document Version}

Publisher's PDF, also known as Version of record

\section{General rights}

Copyright for the publications made accessible via Ulster University's Research Portal is retained by the author(s) and / or other copyright owners and it is a condition of accessing these publications that users recognise and abide by the legal requirements associated with these rights.

\section{Take down policy}

The Research Portal is Ulster University's institutional repository that provides access to Ulster's research outputs. Every effort has been made to ensure that content in the Research Portal does not infringe any person's rights, or applicable UK laws. If you discover content in the Research Portal that you believe breaches copyright or violates any law, please contact pure-support@ulster.ac.uk. 


\title{
Loss of Prolyl Hydroxylase-1 Protects Against Colitis Through Reduced Epithelial Cell Apoptosis and Increased Barrier Function
}

\author{
MURTAZA M. TAMBUWALA, ${ }^{*}$ EOIN P. CUMMINS, ${ }^{*}$ COLIN R. LENIHAN,${ }^{*}$ JUDIT KISS,,${ }^{\ddagger}$ MARKUS STAUCH, ${ }^{\ddagger}$ \\ CARSTEN C. SCHOLZ, ${ }^{*}$ PETER FRAISL, ${ }^{\S}$ FELIX LASITSCHKA," MARTIN MOLLENHAUER, ${ }^{\ddagger}$ SEAN P. SAUNDERS," \\ PATRICK H. MAXWELL," PETER CARMELIET, ${ }^{\S}$ PADRAIC G. FALLON, " MARTIN SCHNEIDER, ${ }^{\ddagger}$ and CORMAC T. TAYLOR* \\ ${ }^{*}$ The Conway Institute, University College Dublin, Ireland; ${ }^{\ddagger}$ Department of General, Visceral and Transplantation Surgery, University of Heidelberg, Germany; ${ }^{\S}$ Vesalius \\ Research Center, VIB, Katholiek University of Leuven, Belgium; "Institute of Pathology, University Hospital, Heidelberg, Germany; "Institute of Molecular Medicine, \\ Trinity College, Dublin, Ireland; and "Rayne Institute, University College, London, United Kingdom
}

BACKGROUND \& AIMS: Hypoxia inducible factor (HIF) prolyl hydroxylase inhibitors are protective in mouse models of inflammatory bowel disease (IBD). Here, we investigated the therapeutic target(s) and mechanism(s) involved. METHODS: The effect of genetic deletion of individual HIF-prolyl hydroxylase (PHD) enzymes on the development of dextran sulphate sodium (DSS)-induced colitis was examined in mice. RESULTS: $\mathrm{PHD}^{-/-}$, but not $\mathrm{PHD}^{+/-}$or $\mathrm{PHD}^{-/-}$, mice were less susceptible to the development of colitis than wild-type controls as determined by weight loss, disease activity, colon histology, neutrophil infiltration, and cytokine expression. Reduced susceptibility of PHD1 $1^{-/-}$mice to colitis was associated with increased density of colonic epithelial cells relative to wild-type controls, which was because of decreased levels of apoptosis that resulted in enhanced epithelial barrier function. Furthermore, with the use of cultured epithelial cells it was confirmed that hydroxylase inhibition reversed DSS-induced apoptosis and barrier dysfunction. Finally, PHD1 levels were increased with disease severity in intestinal tissue from patients with IBD and in colonic tissues from DSS-treated mice. CONCLUSIONS: These results imply a role for PHD1 as a positive regulator of intestinal epithelial cell apoptosis in the inflamed colon. Genetic loss of PHD1 is protective against colitis through decreased epithelial cell apoptosis and consequent enhancement of intestinal epithelial barrier function. Thus, targeted PHD1 inhibition may represent a new therapeutic approach in IBD.

Keywords: Colitis; Hydroxylase; Apoptosis; Hypoxia.

P rolyl hydroxylases (PHDs) are members of a large family of dioxygenases, a subset of which play a key role in intracellular oxygen-sensing and signaling responses during hypoxia, primarily by the regulation of the stability of the hypoxia inducible factor (HIF). ${ }^{1-4}$ Three PHD enzymes that confer oxygen sensitivity to the HIF pathway have been described (PHD1-3)..$^{5,6}$ Although these isoforms share similar biochemical characteristics, they have distinct tissue-specific expression profiles and, as well as regulating HIF, can also affect prosurvival signaling pathways, including nuclear factor $\kappa \mathrm{B}, 1,7-10$

PHDs mediate their effects on HIF through (oxygendependent) hydroxylation of key proline residues on the oxygen-dependent degradation domain of HIF $\alpha$ subunits. When hydroxylated at these sites, $\operatorname{HIF} \alpha$ becomes a target for ubiquitylation by the von Hipple Lindau E3 ubiquitin ligase leading to HIF $\alpha$ ubiquitination and degradation. The specific targets for PHDs in the NF- $\kappa \mathrm{B}$ pathway remain to be determined, although IKK $\alpha$ and IKK $\beta$ contain surface-expressed consensus sites in their regulatory domains. ${ }^{11}$ PHDs play a central role in the adaptive response to hypoxia through activation of these pathways with the subsequent expression of genes promoting cell survival, erythropoiesis, angiogenesis, and metabolism. As such, PHDs may be an effective target for therapeutic manipulation. ${ }^{1,12}$ It has recently become clear that hydroxylase activity can affect the development of inflammation and that hydroxylase inhibitors may represent a novel therapeutic approach in chronic inflammatory disease. ${ }^{12,13}$

Inflammatory bowel disease (IBD) is a genetically heterogenous, chronic inflammatory condition with severe pathology and limited therapeutic options. ${ }^{14-16} \mathrm{Al}-$ though the underlying causes of IBD remain largely undefined, the fundamental defect involves a loss of intestinal epithelial barrier function. We and others have shown that pan-hydroxylase inhibitors such as dimethyloxalylglycine (DMOG) have a protective effect in murine models of colitis and thus represent a potential new therapeutic approach. ${ }^{17,18}$ However, those studies did not

Abbreviations used in this paper: DMOG, dimethyl-oxalylglycine; DSS, dextran sulphate sodium; FITC, fluorescein isothiocyanate; HIF, hypoxia inducible factor; IL-1 $\beta$, interleukin-1 $\beta$; PBS, phosphate-buffered saline; PHD, prolyl hydroxylase; siRNA, short interfering RNA TEER, transepithelial electrical resistance; TUNEL, terminal deoxynucleotidyl transferase-mediated deoxyuridine triphosphate nick-end labeling.

(c) 2010 by the AGA Institute

$0016-5085 / \$ 36.00$

doi:10.1053/j.gastro.2010.06.068 
identify which hydroxylase isoforms or effector pathways are involved.

Mice lacking individual PHD enzymes have distinct phenotypes. PHD1 ${ }^{-1-}$ mice show a reprogrammed basal metabolic profile that decreases muscle performance in healthy tissue but provides acute protection in muscle and liver ischemia. ${ }^{19,20} \mathrm{PHD}^{-/-}$mice are embryonic lethal with a massive vascular defect during embryogenesis, ${ }^{21}$ whereas heterozygous $\left({ }^{+/}\right)$mice show enhanced tumor angiogenesis but decreased metastatic events through what has been termed "endothelial normalization." $22,23 \mathrm{PHD}^{-/-}$mice have reduced neuronal apoptosis, abnormal sympathoadrenal development, and reduced blood pressure. ${ }^{24}$ The diverse phenotypes in mice with different PHD gene deletions strongly suggest that there are distinct isoform-specific functions for the various PHD enzymes in vivo.

To develop our understanding of the mechanisms of protection of pan-hydroxylase inhibitors in colitis, we investigated disease development in PHD1-, PHD2-, and PHD3-deficient mice exposed to dextran sulphate sodium (DSS) in their drinking water to induce colitis. We found that $\mathrm{PHD}^{-/-}$, but not $\mathrm{PHD} 2^{+/-}$or $\mathrm{PHD}^{-/-}$, mice, were selectively protected against the development of colitis. This protection was associated with enhanced intestinal epithelial barrier function which was due to diminished epithelial cell apoptosis. These data implicate a positive role for PHD1 in the regulation of intestinal epithelial cell apoptosis during intestinal inflammation and strengthen the case for the development of PHD1specific inhibitors targeted to intestinal epithelial cells as a new therapeutic approach in the treatment of IBD.

\section{Materials and Methods}

\section{Animals}

$\mathrm{PHD}^{-/-}, \mathrm{PHD}^{-/+}$, and $\mathrm{PHD} 3^{-/-}$mice against a mixed background strain of Swiss/129, which were used for these experiments have been previously described. ${ }^{19,23,24}$ None of the PHD knockout mice showed differential weight gain compared with wild-type mice.

\section{DSS Colitis Model}

The DSS model of colitis was used as described previously. ${ }^{17}$ Briefly, acute disease was induced by the treatment of mice with $5 \%$ DSS in their drinking water for $\leq 6$ days. The disease activity index was determined according to the parameters outlined in Supplementary Table $1 .{ }^{25,26}$ On termination of the experiment, mice were killed by cervical dislocation, and colonic tissues were dissected for further analysis. All procedures described were approved by the relevant institutional Animal Research Ethics Committee.

\section{Assessment of Tissue Inflammation}

Small (approximately $1 \mathrm{~cm}$ ) sections of excised colonic tissue were fixed in 10\% paraformaldehyde $(\mathrm{pH}$,
7.4; phosphate-buffered saline [PBS] buffered) and embedded in paraffin. Sections $(4 \mu \mathrm{m})$ were cut and stained with H\&E. Histologic assessment of colonic mucosae was carried out in a blinded fashion as described in Supplementary Table $2 .{ }^{26}$ To assay for markers of inflammation, colonic tissues were homogenized, and levels of tumor necrosis factor- $\alpha$, interleukin- $1 \beta$ (IL- $1 \beta$ ), IL- 6 , and myeloperoxidase were measured as described previously. ${ }^{17}$

\section{Western Blotting}

Whole colon tissues were thawed, homogenized, sonicated in standard Western blotting lysis buffer, and vigorously stirred until a vortex formed before centrifugation at 12,000 rpm for 5 minutes. Supernatant was removed and normalized to $1 \mu \mathrm{g} / \mu \mathrm{L}$ and separated by sodium dodecyl sulfate-polyacrylamide gel electrophoresis as described previously. ${ }^{17}$ After transfer to nitrocellulose membranes, PHD1 was detected by Western blot with the use of an anti-PHD1 primary antibody (Novus Biologicals, Littleton, CO).

\section{In Vivo Permeability Assay}

Mice were exposed to 6 days of DSS treatment before oral administration of $0.6 \mathrm{mg} / \mathrm{g}$ of body weight fluorescein isothiocyanate (FITC)-labeled dextran (4 $\mathrm{kDa}$ ) by standard oral gavage. Mice were killed 3.5 hours later, and blood was removed by cardiac puncture. Plasma was separated, and FITC levels in the plasma were determined by fluorometry. The distribution of FITCdextran in sectioned colonic tissue was determined by fluorescence microscopy.

\section{In Vitro Permeability Assay}

CaCo-2 epithelial cells (passage 19-26) were seeded on uncoated $6.5-\mathrm{mm}$ semipermeable $(3.0-\mu \mathrm{m}$ pore) Transwell polycarbonate membranes (Costar, Cambridge MA). Monolayers were maintained for 4-5 weeks before experimentation to ensure an efficient barrier and development of transepithelial electrical resistance (TEER). Cells were cotreated with DMOG $1 \mathrm{mmol} / \mathrm{L}$ (or dimethyl sulfoxide vehicle) and 4\% DSS (wt/vol; in Dulbecco's modified Eagle medium supplemented with 10\% fetal calf serum and penicillin/streptomycin) in the apical and basolateral compartments of the Transwell membrane. TEER values were obtained with the use of a REMS Autosampler (World Precision Instruments, Sarasota, FL) at the indicated time points. Values are indicative of triplicate wells per treatment for 4 independent passage numbers.

\section{Immunobistochemistry}

Sections $(4 \mu \mathrm{m})$ were cut from the paraffin blocks, deparaffinized with xylene, and rehydrated in a graded series of alcohols. For immunohistochemical staining, endogenous peroxidase activity was quenched with $3 \%$ hydroxen peroxide solution for 10 minutes. Antigen re- 
trieval was performed by immersing the slides in proteinase $\mathrm{K}$ solution $(20 \mu \mathrm{g} / \mathrm{mL}$ in $10 \mathrm{mmol} / \mathrm{L}$ Tris- $\mathrm{HCl}, \mathrm{pH}$ 7.5) for 12 minutes. Sections were blocked with $5 \%$ goat serum for 1 hour at room temperature and then incubated overnight with primary antibody at $4^{\circ} \mathrm{C}$ in a humidified chamber. The primary antibodies used were rabbit anti-KI-67 (1:100 dilution; Abcam, Cambridge, United Kingdom), rabbit anti-cleaved caspase-3 (Asp175; 1:200 dilution; Cell Signaling Technology, Danvers, MA), and rabbit anti-cytokeratin 8 (1:100 dilution; Abcam). The slides were then treated with biotinylated goat antirabbit antibody (1:200 dilution; Vector Laboratories, Peterborough, United Kingdom). ABC immunodetection (VECTASTAIN Elite ABC Kit; Vector Laboratories) was used to detect cleaved caspase 3 and KI-67. The sections were developed with diaminobenzidine as a chromagen and counterstained with hematoxylin. Cytokeratin 8 was detected with avidin-conjugated Texas red (1:50 dilution; Merck Biosciences, Darmstadt, Germany) followed by nuclear counterstain with Hoechst 33342 dye. PBS/1\% Tween solution was used to wash sections between steps. Colonic sections from 5 to 7 animals were stained for $\mathrm{KI}-67$, cytokeratin 8 , or cleaved caspase 3 . In the case of KI-67 and cleaved caspase 3, for each section, three $200 \times$ or $400 \times$ fields, respectively, were randomly selected, and the number of positive cells per 100 enterocytes was quantified. All scoring was carried out in a blinded fashion.

\section{Terminal Deoxynucleotidyl Transferase-Mediated Deoxyuridine Triphosphate Nick-End Labeling Stain}

Cellular apoptosis was identified with terminal deoxynucleotidyl transferase-mediated deoxyuridine triphosphate nick-end labeling (TUNEL; Fluorescein In Situ Cell Death Detection Kit; Roche Diagnostic, Mannheim, Germany). After de-waxing, rehydration and proteinase $\mathrm{K}$ antigen retrieval colon sections were incubated with the TUNEL reaction mix for 1 hour at $37^{\circ} \mathrm{C}$ in a humidified chamber. Sections were counterstained with Hoechst dye and analyzed with fluorescent microscopy. Colonic sections from 5 animals per group were TUNEL stained. For each section, three $400 \times$ fields were randomly selected, and the number of positive cells per 100 enterocytes was quantified. All scoring was carried in a blinded fashion.

\section{In Vitro Apoptosis Assay}

CaCo- 2 cells were treated with DMOG $(1 \mathrm{mmol} / \mathrm{L}$, 24 hours) or transfected with $50 \mathrm{nmol} / \mathrm{L}$ PHD1 short interfering RNA (siRNA) or control nontarget siRNA (Dharmacon, Lafayette, CO). Transfections were carried out with Lipofectamine 2000 (Invitrogen, Paisley, United Kingdom). After treatment, cells were treated with DSS (1\%, 24 hours). The cells were harvested, resuspended in ice-cold PBS, and incubated with YO-PRO-1, Hoechst 33342, and propridium iodide (Vybrant Apoptosis Assay
Kit \#7; Invitrogen) according to the manufacturer's protocol. Flow cytometric analyses were carried out with the CyAn ADP analyser (Beckman Coulter, Fullerton, CA) and Summit 4.3 software. Cells were defined as living, early apoptotic, or late apoptotic/necrotic according to their relative dye uptake.

\section{Statistical Analysis}

Statistical comparisons were made by analysis of variance or Student $t$ test. Values are expressed as mean \pm SEM for $n$ individual experiments.

\section{Results \\ PHD1-Deficient Mice Are Protected Against DSS-Induced Colitis}

Previous studies have shown that treatment with pan-hydroxylase inhibitors is protective in DSS-induced colitis in mice. ${ }^{17,18}$ Those studies used broad-spectrum hydroxylase inhibitors such as DMOG because pharmacologic isoform-specific inhibitors are currently unavailable. To determine which PHD enzyme or multiple enzymes are responsible for the protective effects of panhydroxylase inhibition, we investigated the development of DSS-induced colitis in $\mathrm{PHD}^{-/-}, \mathrm{PHD}^{+/-}$, and $\mathrm{PHD}^{-/-}$mice. We found that only PHD $1^{-/-}$mice were significantly protected from DSS-induced colitis as determined by weight loss (Figure $1 A$ ) and disease activity index measurements (Figure $1 B$ ). PHD1 ${ }^{-/-}$mice also had significantly attenuated histologic signs of inflammation compared with wild-type controls (Figure $1 C$ and $D$ ). The reduced severity of DSS-induced colitis in $\mathrm{PHD}^{-/-}$mice was confirmed by the significantly lower levels of myeloperoxidase enzymatic activity, a marker of neutrophil infiltration, in the colons of PHD1 $1^{-/-}$mice relative to wild-type animals (Figure 1E). Furthermore, the levels of DSS-induced inflammatory cytokines, including IL- $1 \beta$, tumor necrosis factor- $\alpha$ and IL-6, were significantly lower in PHD $1^{-/-}$mice than in wild-type controls (Figure $1 E$ ). In contrast, $\mathrm{PHD}^{+/-}$and $\mathrm{PHD}^{-/-}$mice did not show attenuated DDS-induced inflammation in all parameters investigated (data not shown). Taken together, these data indicate that $\mathrm{PHD}^{-/-}$mice are selectively protected against the development of colitis.

\section{Increased Enterocyte Density in PHD-Deficient Mice in Colitis}

We next carried out high magnification $(400 \times)$ microscopic analysis of mucosal structure in wild-type and PHD $1^{-/-}$mice exposed to DSS. Increased intestinal epithelial cell density was observed in $\mathrm{PHD}^{-/-}$mice treated with DSS compared with wild-type controls (Figure $2 A$ ). The insets in Figure $2 A$ represent high magnification areas of intestinal epithelial cells showing increased epithelial density in colonic tissues derived from DSS-treated $\mathrm{PHD}^{-/-}$mice compared with wild-type mice. The enhanced epithelium was confirmed by epithe- 
A
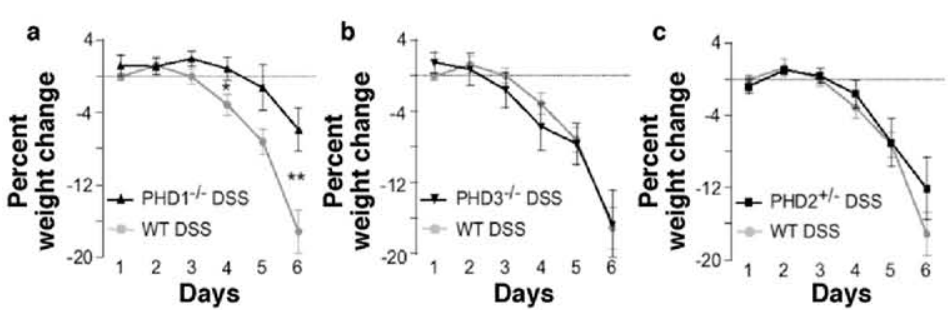

B
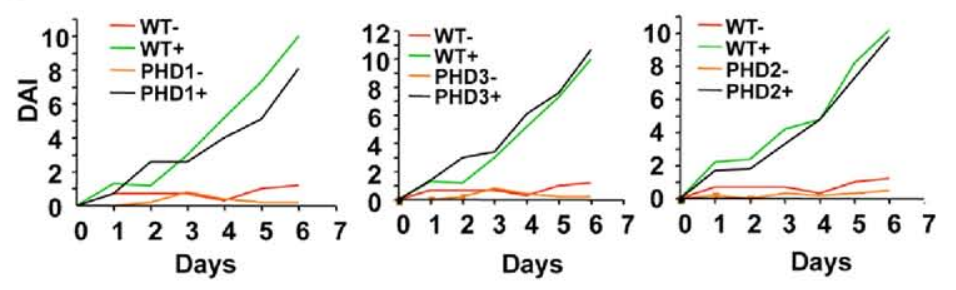

D

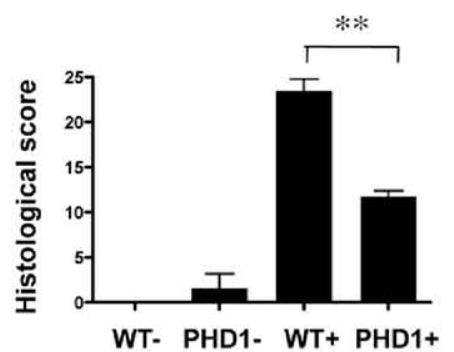

C

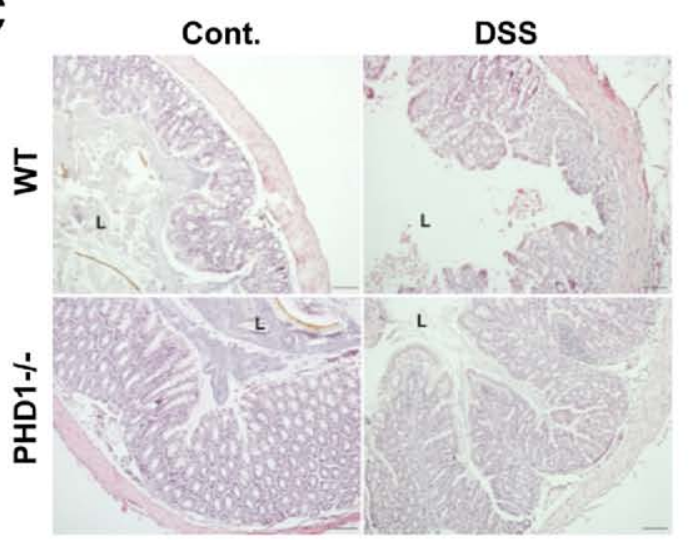

E
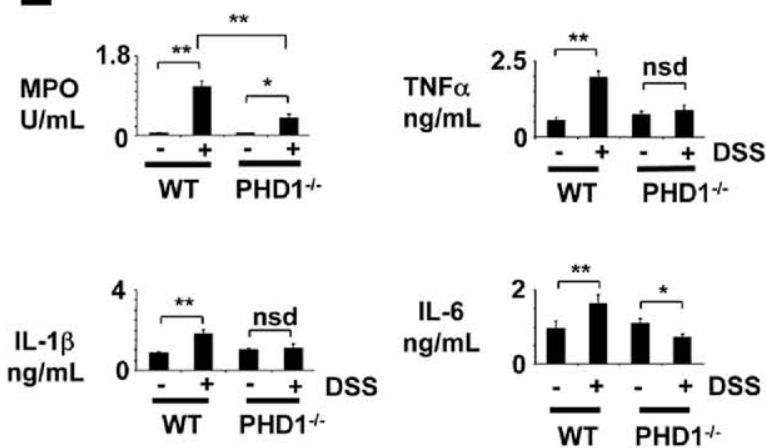

Figure 1. PHD1 $1^{-1}$ mice are selectively protected against DSS-induced colitis. (A) Weight change measurements over 6 days in wild-type (WT), $\mathrm{PHD1}^{-1-}$, $\mathrm{PHD}^{+/-}$, or $\mathrm{PHD3}^{-/-}$mice exposed to $5 \% \mathrm{DSS}$. (B) Disease activity indices over 6 days in wild-type, $\mathrm{PHD1}^{-/-}$, $\mathrm{PHD2}^{+/-}$, or PHD3 ${ }^{-/-}$ mice exposed to DSS (+). (C) Representative 100 $\times$ histologic images and (D) histologic scores of colonic tissue taken from control and DSS-treated wild-type or PHD1 ${ }^{-1-}$ mice (scale bar $\left.=100 \mu \mathrm{m}\right)$. (E) Myeloperoxidase $(\mathrm{MPO})$, tumor necrosis factor- $\alpha(\mathrm{TNF} \alpha)$, IL-1 $\beta$, and IL-6 levels were determined in wild-type and $\mathrm{PHD1}^{-/-}$mouse colonic tissue 6 days after initiation of administrating DSS. Each control and experimental group contained a minimum of 6 individual mice. ${ }^{\star} P<.05 ;{ }^{* *} P<.01 ;$ nsd, not significantly different; L, lumen.

lial-specific cytokeratin staining in colonic tissues (Figure $2 B)$, which shows decreased ulceration in DSS-treated PHD1 ${ }^{-/-}$mice compared with wild-type controls. Collectively, these data led us to hypothesize that the protective effects of PHD1 deficiency were due to enhanced epithelial barrier function as a result of increased intestinal epithelial cell density.

\section{Enhanced Epithelial Barrier Function in PHD1 $^{-/-}$Mice}

To further address the effects of loss of PHD1 on the intestinal epithelial barrier, in vivo intestinal barrier function was measured in wild-type and $\mathrm{PHD} 1^{-/-}$mice exposed to DSS. Mice were given an oral dose of FITCdextran on the final day of DSS exposure, and 4 hours later FITC levels in the plasma were determined as a measure of intestinal permeability. Although DSS induced a characteristic increase in intestinal permeability as reflected by increased appearance of FITC in plasma, this was markedly diminished in $\mathrm{PHD} 1^{-/-}$mice (Figure $3 A$ ). Furthermore, fluorescent microscopic detection of
FITC in colonic tissues from these animals showed retention of FITC at the epithelial barrier in PHD1-/mice, whereas in wild-type mice FITC had transcended the epithelial barrier (Figure 3B).

To test whether hydroxylase inhibition can directly affect epithelial barrier function, we next exposed cultured intestinal epithelial cells $(\mathrm{CaCo}-2)$, grown on permeable support inserts to increasing periods of DSS and measured barrier disruption by measuring TEER. Cells treated with the hydroxylase inhibitor DMOG showed reduced barrier dysfunction compared with cells treated with vehicle and DSS alone, indicating that hydroxylase inhibition in isolated enterocytes confers resistance to DSS-induced barrier dysfunction (Supplementary Figure 1). Taken together, these data indicate that loss of PHD1 leads to enhanced epithelial barrier function in colitis.

\section{Decreased Enterocyte Apoptosis in $\mathrm{PHD}^{-/-}$ Mice Exposed to DSS}

We next investigated whether the increase in enterocyte density (Figure 2) and enhanced barrier function 
A

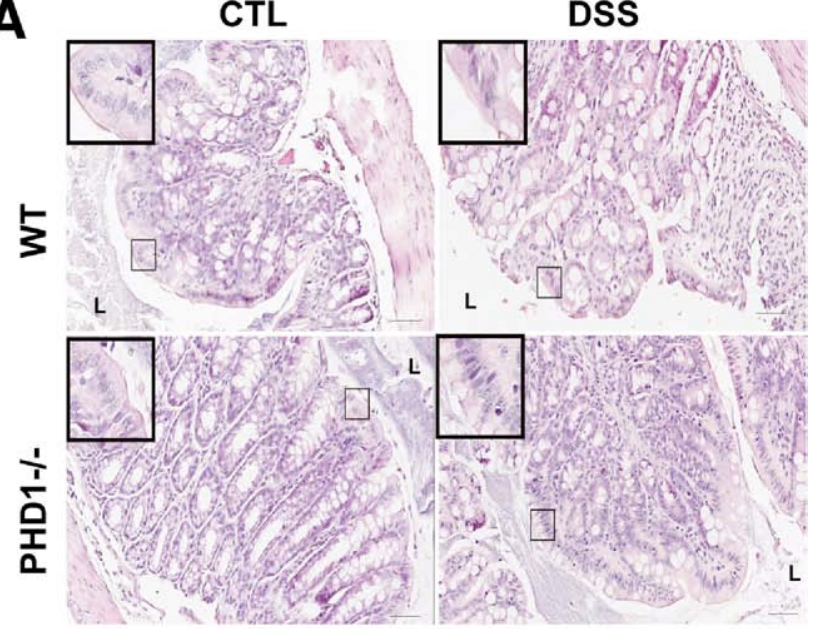

B

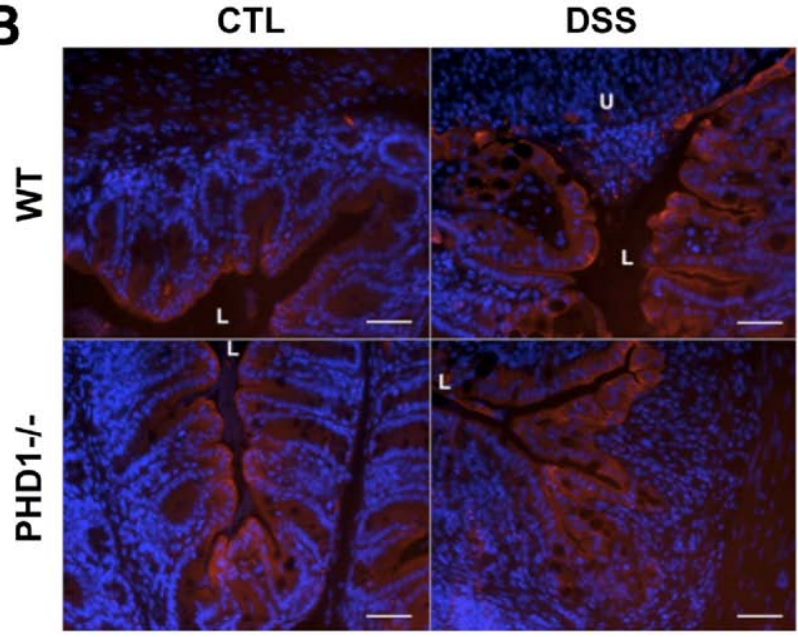

Figure 2. Increased epithelial density in homozygous PHD1 knockout mice exposed to DSS. (A) High magnification (400X) histologic images of colonic tissue from wild-type (WT) and PHD1 ${ }^{-1-}$ mice with and without DSS treatment shows increased intestinal epithelial density in tissues from PHD1 ${ }^{-1-}$ animals (scale bar $=25 \mu \mathrm{m}$ ). Insets show examples of areas of epithelium in high magnification (1600X). L, lumen; $U$, region of ulceration. (B) Immunohistochemical analysis (200X) of cytokeratin (staining red) and nuclei (staining blue) in colonic tissue from wild-type and PHD1 ${ }^{-/-}$mice with DSS treatment shows increased mucosal ulceration and decreased epithelial density in wild-type mice compared with PHD1 ${ }^{-1-}$ mice (scale bar $=50 \mu \mathrm{m}$ ). Each control and experimental group contained a minimum of 6 individual mice (L, lumen; $U$, area of ulceration).

(Figure 3) observed in $\mathrm{PHD}^{-/-}$mice was due to decreased epithelial cell death or increased proliferation. Immunohistochemical KI67 staining (a marker of proliferating cells) was not different between wild-type and PHD1 ${ }^{-/-}$mice either with or without DSS treatment, indicating no difference in proliferation between groups (Figure 4A).

We next investigated intestinal epithelial cell apoptosis with the use of both TUNEL staining and detection of cleaved caspase 3 . Basal rates of apoptosis detected with both techniques were similar in PHD1 $1^{-/}$and wild-type mice. However, $\mathrm{PHD}^{-/-}$mice exhibited a significantly lower degree of enterocyte apoptosis when exposed to DSS than did wild-type mice (Figure $4 B$ and $C$ ). Collectively, these data indicate that $\mathrm{PHD} 1^{-/-}$mice are resistant to DSS-induced colitis because of barrier-protective effects associated with decreased enterocyte apoptosis.

To investigate whether PHD1 inhibition could directly affect epithelial apoptosis, cultured enterocytes were exposed to DSS in the presence and absence of the pan hydroxylase inhibitor DMOG or siRNA targeted to PHD1, and the induction of early and late apoptosis was measured. DMOG (Figure 5A) or PHD1 siRNA (Figure $5 B$ ) significantly inhibited DSS-induced apoptosis in isolated enterocytes, indicating a direct antiapoptotic effect of pharmacologic hydroxylase inhibition on epithelial cells.
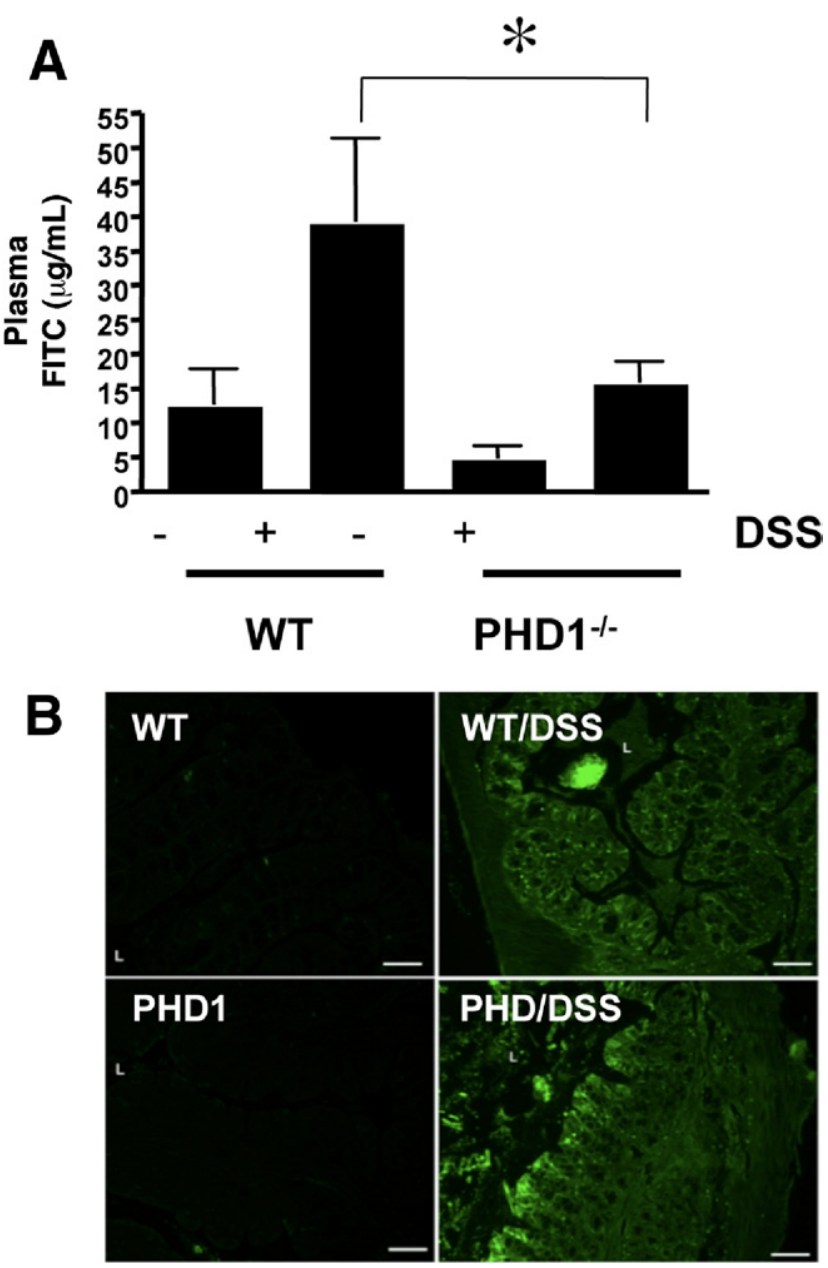

Figure 3. Enhanced epithelial barrier function in $\mathrm{PHD}^{-1-}$ mice. (A) Intestinal permeability was measured by the appearance of orally administered FITC-labeled dextran in plasma from wild-type and PHD1 ${ }^{-1-}$ mice exposed to DSS (bars represent mean \pm SEM; ${ }^{*} P<.5$; $n=6)$. (B) Fluorescence microscopy $(100 \times)$ of the intestinal mucosa from DSS-treated wild-type (WT) and $\mathrm{PHD1}^{-1-}$ mice exposed to orally administered FITC-labeled dextran shows FITC permeation into tissue is enhanced in wild-type animals treated with DSS compared with $\mathrm{PHD}^{-/-}$animals whereby FITC-dextran is retained at the epithelium (scale bar $=100 \mu \mathrm{m}) \mathrm{L}$, lumen. 
A CTL

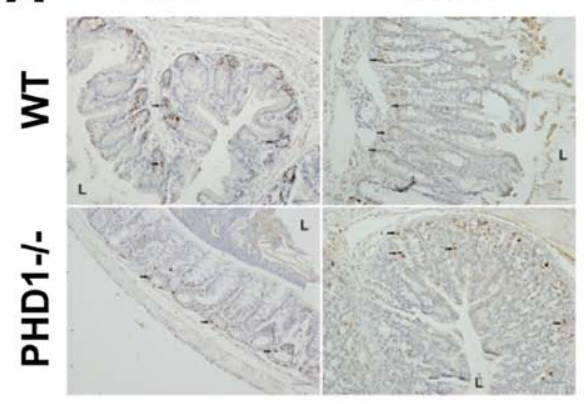

B CTL

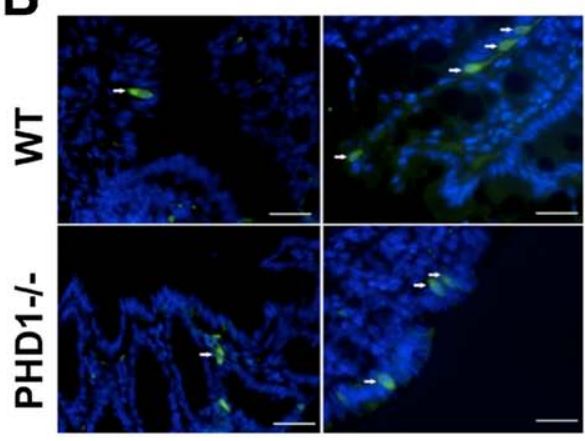

胥

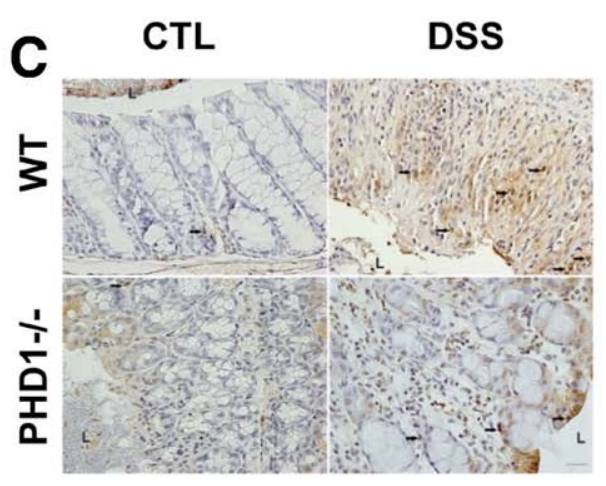

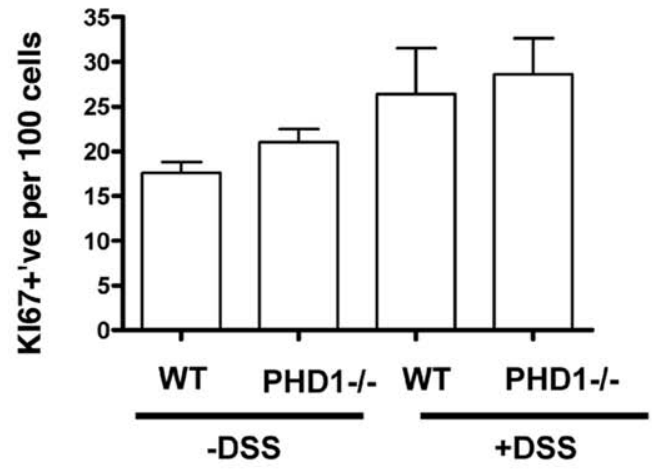
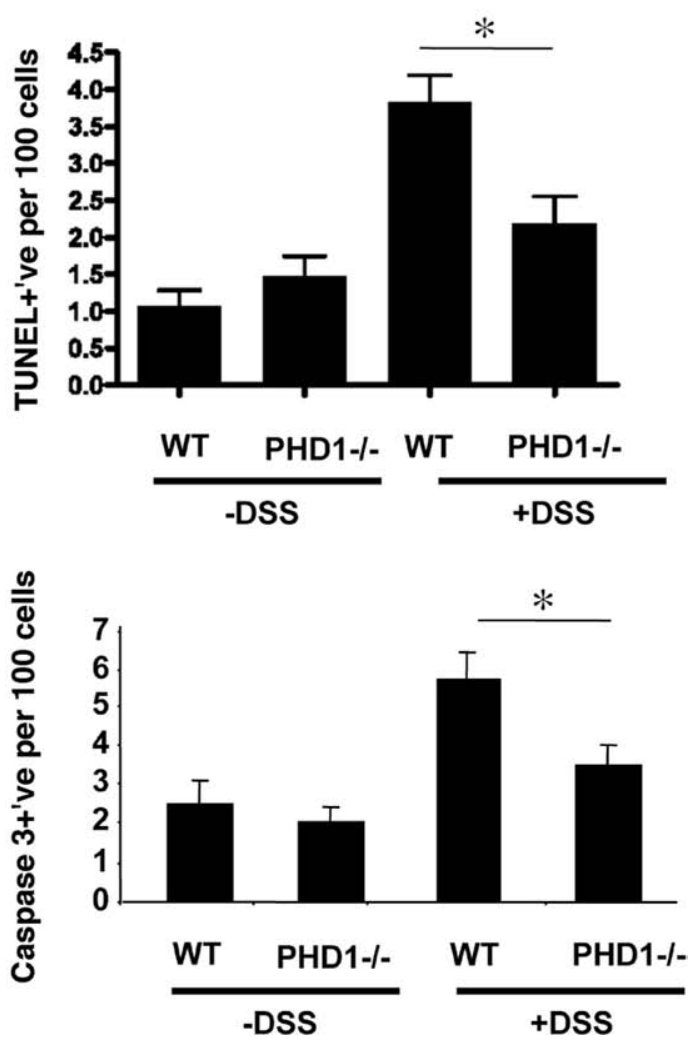

Figure 4. Decreased enterocyte apoptosis in $\mathrm{PHD1}^{-/-}$mice exposed to DSS. (A) Representative 100x immunohistochemical images (left) and quantification of intestinal epithelial cell proliferation (right) in wild-type (WT) and $\mathrm{PHD}^{-1-}$ mice exposed to DSS as measured by $\mathrm{KI}-67$ staining (scale bar = $50 \mu \mathrm{m})$. (B) Representative 100x immunofluorescence images (left) and quantification of intestinal epithelial cell apoptosis (right) in wild-type and $\mathrm{PHD1}^{-1-}$ mice exposed to DSS as measured by TUNEL staining (scale bar $=50 \mu \mathrm{m})$. (C) Representative 200X immunohistochemical images (left) and quantification of intestinal epithelial cell apoptosis (right) in wild-type and $\mathrm{PHD}^{-1-}$ mice exposed to DSS as measured by cleaved caspase-3 staining (scale bar $=$ $25 \mu \mathrm{m})$. Each control and experimental group contained a minimum of 6 individual mice. ${ }^{\star} P<$ .05 .

\section{PHD1 Expression Is Correlated With Disease Severity in Patients With IBD}

Having identified PHD1 as a potential target for pharmacologic intervention in IBD, we next examined the expression of PHD1 in intestinal tissue taken from patients undergoing restorative proctocolectomy for surgical treatment of IBD. Intestinal tissues from patients with IBD were independently scored by 2 clinical pathologists and categorized for histopathologic grade of inflammation according to the Truelove and Richards scale $^{27}$ (grade 0 , no inflammation; grade 1 , chronic inactive inflammation [increased content of mononuclear cells within the mucosal lamina propria]; grade 2, mild active inflammation [plus few circumscript foci with neutrophilic intraepithelial infiltrates/cryptitis or single crypt abscesses]; grade 3 , moderate active inflammation [plus several foci with neutrophilic intraepithelial infiltrates/cryptitis or crypt abscesses]). Semiquantitative analysis of immunoblots showed significantly higher expression of PHD1 protein in colon samples that displayed active mucosal inflammation (Figure 6A and $B$ ). Indeed, PHD1 was relatively overexpressed in $100 \%$ of tissue samples displaying high inflammatory activity (Truelove and Richards score 2-3). By contrast, relative overexpression of PHD1 was only observed in $33 \%$ of samples with inactive inflammation (Truelove and Richards score $0-1$ ). This difference was significant when applying the Fisher's exact test $(P=.015$; $\mathrm{n}=$ 8). Similarly, colonic tissue from mice showed increased PHD1 expression after DSS exposure (Supplementary Figure 2). In summary, although PHD deficiency or inhibition is associated with decreased disease activity, increased PHD expression is associated with disease severity. 


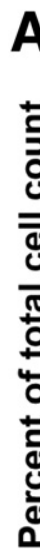

A

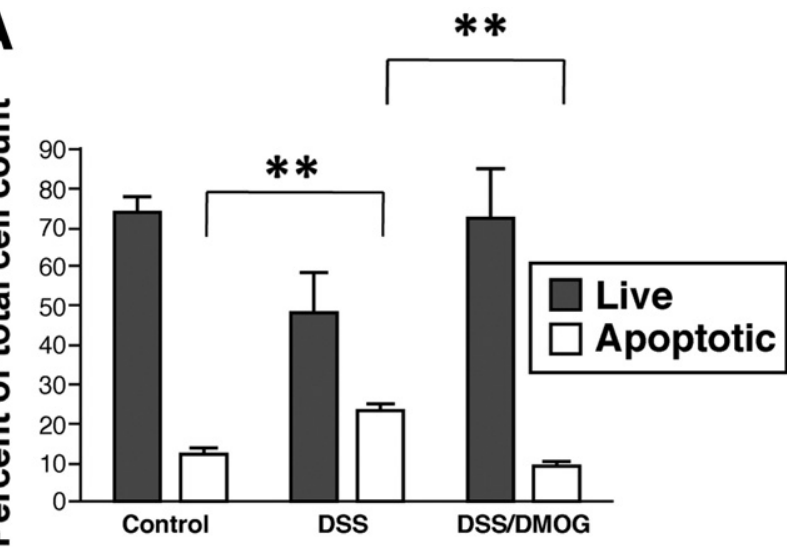

B
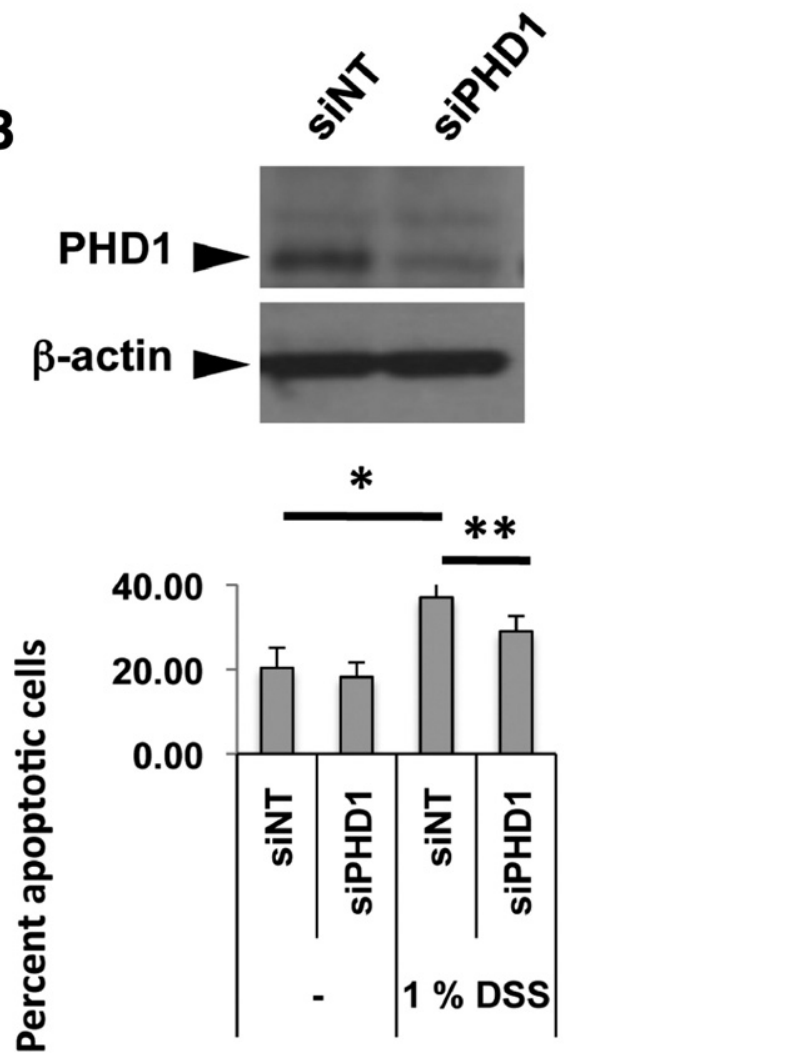

Figure 5. PHD1 inhibition blocks apoptosis in cultured enterocytes. (A) CaCo-2 intestinal epithelial cells were exposed to 1\% DSS or vehicle for 24 hours with and without pretreatment with DMOG $(1 \mathrm{mmol} / \mathrm{L})$. Numbers of live and apoptotic epithelial cells were determined by flow cytometric analysis. (B) CaCo-2 cells were exposed to siRNA directed against PHD1. Specific siRNA treatment resulted in effective knockdown of PHD1 (top) and resulted in decreased numbers of DSS-induced apoptotic cells (bottom). Data shown reflect a minimum of 4 individual experiments. ${ }^{\star} P<.05 ;{ }^{\star \star} P<.01$.

\section{Discussion}

A common feature of all IBD regardless of the underlying cause is the loss of intestinal epithelial barrier function because of excessive epithelial cell death, an event that allows the nonspecific movement of luminal antigenic material into the submucosa. ${ }^{28}$ The resulting inflammatory response drives further barrier dysfunction and the symptoms associated with the disease, thus establishing a positive feed forward loop between developing inflammation and barrier dysfunction. The intestinal epithelial barrier relies heavily on the controlled turnover of intestinal epithelial cells, which originate from stem cells in the intestinal crypts and migrate to the top of the crypt or villus before undergoing apoptosis and being released into the intestinal lumen. The regulation of the intestinal epithelial cell turnover is a complex balance between epithelial proliferation and cell death in which stem cells play a key role. ${ }^{29}$ In the physiologic state, intestinal epithelial cells have a relatively short life span, and their programmed cell death through apoptosis is a carefully controlled process that is probably critical for the maintenance of normal barrier function. ${ }^{30}$ The regulatory factors that are responsible for the initiation of physiologic intestinal epithelial cell apoptosis remain to be fully elucidated; however, increased rates of apoptosis with resultant dysfunction of the intestinal epithelial barrier is central to the pathology of IBD. ${ }^{31}$ Thus, therapies directed toward delaying or suppressing intestinal epithelial cell apoptosis may allow time for mucosal healing to occur in IBD and may thus represent a therapeutic approach.

We, and others, have demonstrated that pan hydroxylase inhibition is protective against the development of
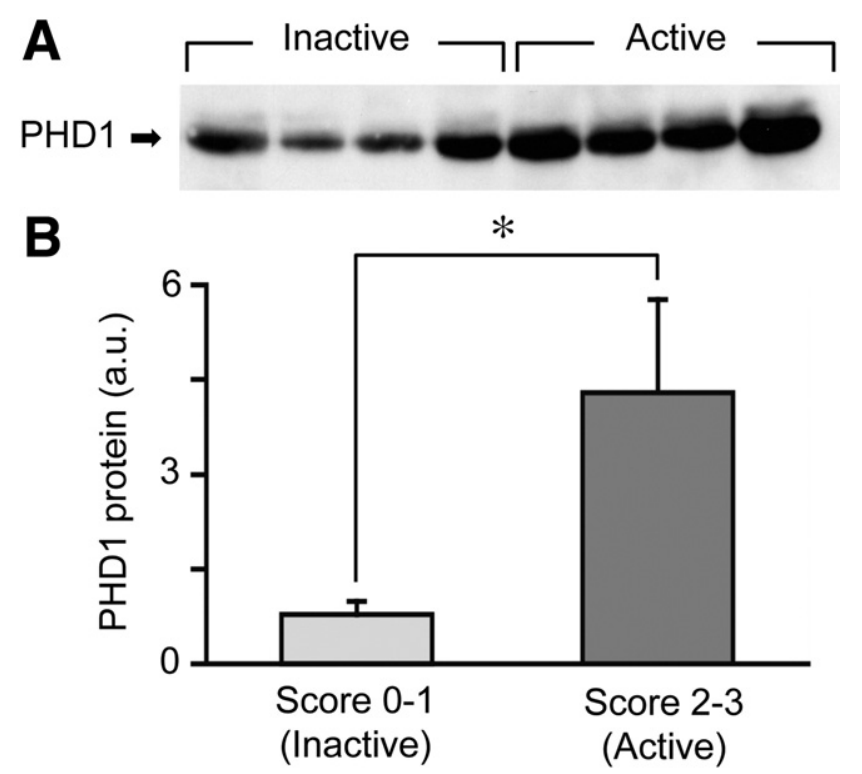

\section{Histological grade of inflammation}

Figure 6. PHD1 levels correlate with inflammatory score in patients with IBD. (A) Representative immunoblots of colon tissues from patients with ulcerative colitis with either inactive (left) or active (right) mucosal inflammation. Equal amounts of protein were loaded. (B) Densitometric analysis of PHD1 protein concentrations in colon mucosa from patients with inactive (Truelove and Richards score $0-1$ ) or active mucosal inflammation (score 2-3), normalized to the expression of $\beta$-actin. Bars represent mean \pm SEM of relative band intensity values (a.u., arbitrary units). ${ }^{*} P<.05 ; \mathrm{n}=8$. 
colitis in multiple models. ${ }^{17,18}$ In the current study, we find that PHD1 ${ }^{-/-}$mice selectively show a significant decrease in DSS-induced apoptosis, an event that we hypothesize to be linked to enhanced barrier function and subsequent protection against the development of colitis. This is supported by studies that investigated the link between hydroxylase activity and apoptosis in other model systems, which have shown an antiapoptotic effect of pharmacologic hydroxylase inhibition..$^{32-34}$

Hydroxylase inhibition affects multiple transcriptional pathways, including HIF and NF- $\kappa$ B, both of which have been shown to be protective in epithelial cells during IBD. ${ }^{35} \mathrm{HIF}$ activates the expression of a range of factors, including intestinal trefoil factor, CD73, and the adenosine $\mathrm{A} 2 \mathrm{~B}$ receptor that have been shown to be barrier protective. ${ }^{35}$ Furthermore, NF- $\kappa \mathrm{B}$ activation results in increased expression of antiapoptotic factors. ${ }^{36}$ We have proposed that the combined effects of barrier protective and antiapoptotic gene expression in epithelial cells, which occurs by these pathways, underpin the effectiveness of pan-hydroxylase inhibitors in models of IBD. ${ }^{35} \mathrm{In}$ the current study, we have identified that PHD1 is the key target hydroxylase in mediating the protective effects of hydroxylase inhibitors in colitis and thus represents a likely therapeutic target for IBD. Importantly, because of the lethality of a homozygous PHD2 knockout mouse, we cannot rule out a possible role for a protective effect of complete PHD2 inhibition during colitis. Future studies will determine whether the protection afforded by hydroxylase inhibition is by activation of HIF, NF- $\kappa \mathrm{B}$, or other transcriptional regulators. Increased PHD1 correlates with disease severity in patients with IBD, reflecting a positive relationship between the expression levels of PHD1 and disease severity. In a previous study, Robinson et $\mathrm{al}^{18}$ demonstrated that PHD isoform mRNA expression levels in the intestinal mucosa were in the order PHD2 $=$ PHD3 $>$ PHD1. Although the mRNA expression levels of PHD1 are lowest in noninflamed tissue, we found that PHD1 levels were increased in inflamed tissues from patients with IBD, raising the intriguing possibility that increased PHD1 expression or activity may be contributing to disease progression through increasing epithelial cell apoptosis and reducing barrier function. In summary, we propose that, as a positive regulator of intestinal epithelial cell apoptosis in colitis, PHD1 represents a new therapeutic target in the treatment of inflammatory bowel disease.

\section{Supplementary Material}

Note: To access the supplementary material accompanying this article, visit the online version of Gastroenterology at www.gastrojournal.org, and at doi: $10.1053 /$ j.gastro.2010.06.068.

\section{References}

1. Kaelin WG Jr, Ratcliffe PJ. Oxygen sensing by metazoans: the central role of the HIF hydroxylase pathway. Mol Cell 2008;30: 393-402.

2. Schipani E, Maes C, Carmeliet G, et al. Regulation of osteogenesis-angiogenesis coupling by HIFs and VEGF. J Bone Miner Res 2009;24:1347-1353.

3. Semenza GL. Involvement of oxygen-sensing pathways in physiologic and pathologic erythropoiesis. Blood 2009;114:20152019.

4. Semenza GL. Hypoxia-inducible factor 1 and cancer pathogenesis. IUBMB Life 2008;60:591-597.

5. Epstein AC, Gleadle JM, McNeill LA, et al. C. elegans EGL-9 and mammalian homologs define a family of dioxygenases that regulate HIF by prolyl hydroxylation. Cell 2001;107:43-54.

6. Ivan M, Haberberger T, Gervasi DC, et al. Biochemical purification and pharmacological inhibition of a mammalian prolyl hydroxylase acting on hypoxia-inducible factor. Proc Natl Acad Sci U S A 2002;99:13459-13464.

7. Cummins EP, Berra E, Comerford KM, et al. Prolyl hydroxylase-1 negatively regulates IkappaB kinase-beta, giving insight into hypoxia-induced NFkappaB activity. Proc Natl Acad Sci U S A 2006; 103:18154-18159.

8. Chan DA, Kawahara TL, Sutphin PD, et al. Tumor vasculature is regulated by PHD2-mediated angiogenesis and bone marrowderived cell recruitment. Cancer Cell 2009;15:527-538.

9. Xue J, Li X, Jiao S, et al. Prolyl hydroxylase-3 is downregulated in colorectal cancer cells and inhibits IKKbeta, independent of hydroxylase activity. Gastroenterology 2010;138:606-615.

10. Fu J, Taubman MB. The prolyl hydroxylase EGLN3 regulates skeletal myoblast differentiation through an NF-kappaB-dependent pathway. Biol Chem 2010;285:8927-8935.

11. Oliver KM, Taylor CT, Cummins EP. Hypoxia. Regulation of NFkappaB during inflammation: the role of hydroxylases. Arthritis Res Ther 2009;11:215-220.

12. Frais $P$, Aragonés J, Carmeliet $P$. Inhibition of oxygen sensors as a therapeutic strategy for ischaemic and inflammatory disease. Nat Rev Drug Discov 2009;8:139-152.

13. Nizet V, Johnson RS. Interdependence of hypoxic and innate immune responses. Nat Rev Immunol 2009;9:609-617.

14. Braus NA, Elliott DE. Advances in the pathogenesis and treatment of IBD. Clin Immunol 2009;132:1-9.

15. Rutgeerts $P$, Vermeire $S$, Van Assche G. Biological therapies for inflammatory bowel diseases. Gastroenterology 2009;136: 1182-1197.

16. Imielinski M, Baldassano RN, Griffiths A, et al. Common varients at five new loci associated with early-onset inflammatory bowel disease. Nature Genet 2009;41:1335-1340.

17. Cummins EP, Seeballuck F, Keely SJ, et al. The hydroxylase inhibitor dimethyloxalylglycine is protective in a murine model of colitis. Gastroenterology 2008;134:156-165.

18. Robinson A, Keely S, Karhausen J, et al. Mucosal protection by hypoxia-inducible factor prolyl hydroxylase inhibition. Gastroenterology 2008;134:145-155.

19. Aragonés J, Schneider M, Van Geyte K, et al. Deficiency or inhibition of oxygen sensor Phd1 induces hypoxia tolerance by reprogramming basal metabolism. Nat Genet 2008;40: 170-180.

20. Schneider M, van Geyte K, Fraisl P, et al. Loss or silencing of the PHD1 prolyl hydroxylase protects livers of mice against ischemia/reperfusion injury. Gastroenterology 2009;138: 3386-3400.

21. Takeda K, Ho VC, Takeda H, et al. Placental but not heart defects are associated with elevated hypoxia inducible factor alpha levels in mice lacking prolyl hydroxylase domain protein 2. Mol Cell Biol 2006;26:8336-8346. 
22. Ozolin TR, Fisher TS, Nadeau DM, et al. Defect in embryonic development of EGLN1/PHD2 knockdown transgenic mice is associated with induction of lgfbp in the placenta. Biochem Biophys Res Commun 2009;390:372-376.

23. Mazzone M, Dettori D, Leite de Oliveira R, et al. Heterozygous deficiency of PHD2 restores tumor oxygenation and inhibits metastasis via endothelial normalization. Cell 2009;136:839-851.

24. Bishop T, Gallagher D, Pascual A, et al. Abnormal sympathoadrenal development and systemic hypotension in PHD3-/- mice. Mol Cell Biol 2008;28:3386-3400.

25. Cooper HS, Murthy SN, Shah RS, et al. Clinicopathologic study of dextran sulfate sodium experimental murine colitis. Lab Invest 1993;69:238-249.

26. Williams KL, Fuller CR, Dieleman LA, et al. Enhanced survival and mucosal repair after dextran sodium sulfate-induced colitis in transgenic mice that overexpress growth hormone. Gastroenterology 2001;120:925-937.

27. Truelove SC, Richards WC. Biopsy studies in ulcerative colitis. $\mathrm{Br}$ Med J 1956;1:1315-1318.

28. Abraham C, Cho JH. Inflammatory bowel disease. N Engl J Med 2009;361:2066-2078.

29. van der Flier LG, Clevers H. Stem cells, self-renewal, and differentiation in the intestinal epithelium. Annu Rev Physiol 2009;71: 241-260.

30. Hall PA, Coates PJ, Ansari B, et al. Regulation of cell number in the mammalian gastrointestinal tract: the importance of apoptosis. J Cell Sci 1994;107:3569-3577.

31. Schulzke JD, Ploeger S, Amasheh M, et al. Epithelial tight junctions in intestinal inflammation. Ann N Y Acad Sci 2009;1165: 294-300.

32. Liu XB, Wang JA, Ogle ME, et al. Prolyl hydroxylase inhibitor dimethyloxalylglycine enhances mesenchymal stem cell survival. J Cell Biochem 2009;106:903-911.

33. Lomb DJ, Straub JA, Freeman RS. Prolyl hydroxylase inhibitors delay neuronal cell death caused by trophic factor deprivation. J Neurochem 2007;103:1897-1906.
34. Nangaku M, Izuhara Y, Takizawa S, et al. A novel class of prolyl hydroxylase inhibitors induces angiogenesis and exerts organ protection against ischemia. Arterioscler Thromb Vasc Biol 2007; 27:2548-2554.

35. Taylor CT, Colgan SP. Hypoxia and gastrointestinal disease. J Mol Med 2007;85:1295-1300.

36. Luo JL, Kamata H, Karin M. The anti-death machinery in IKK/ NF-kB signaling. Clin Immunol 2005;25:541-550.

Received March 9, 2010. Accepted June 21, 2010.

\section{Reprint requests}

Address requests for reprints to: Cormac T. Taylor, PhD, UCD Conway Institute, University College Dublin, Belfield, Dublin 4, Ireland. e-mail: cormac.taylor@ucd.ie; fax: +35317166710.

\section{Acknowledgments}

The authors thank Prof Peter Ratcliffe (Oxford University) who contributed to the generation of the PHD knockout mice, Dimitri Scholz (University College Dublin) for imaging advice, Alfonso Blanco for technical flow cytometric support, and Janet McCormack for technical support for slide scanning.

M.M.T., E.P.C., and C.R.L. contributed equally to this study. M.S. and C.T.T. contributed equally to this study.

\section{Conflicts of interest}

The authors disclose no conflicts.

\section{Funding}

This work was funded by grants from Science Foundation Ireland (to C.T.T.) and the Emmy Noether Program of the German Research Foundation (to M.S.). Further support was provided by EU COST action TD0901 and Systems Biology Ireland. 